\title{
Impact of cocoa on short chain fatty acid production from ispaghula by colonic bacteria in vitro
}

\author{
B. Mansoorian, A. Garcia, E. Combet and C. A. Edwards \\ University of Glasgow, Human Nutrition, Yorkhill hospital, Glasgow G3 8SJ, UK
}

Studies investigating the food matrix interactions between fibre and polyphenols are scarce, especially in humans. In vitro studies have suggested that fermentable carbohydrates can alter the rate of bacterial metabolism of polyphenols and the concentrations of intermediate $^{(1)}$ and final metabolites ${ }^{(2)}$. Several polyphenols have been shown to have antibacterial properties ${ }^{(3,4)}$ which may potentially inhibit carbohydrate fermentation. In animal studies, polyphenolics had little impact on fibre fermentation ${ }^{(5)}$. In this study, the impact of cocoa (C) on fermentation of ispaghula (I, psyllium husk) was investigated. Fresh faecal samples from 10 healthy volunteers (21-34 years) who followed a low polyphenol diet 3 days before sample collection were used to inoculate an in-vitro fermentation model ${ }^{(6)}$. Fibre $(1 \mathrm{~g})$ and dark cocoa ( $0.5 \mathrm{~g}$ extra brute Cocoa-Cacao Barr, Barry Callebaut, Hardricourt, France; $0.13 \mathrm{~g}$ fibre) were added to $50 \mathrm{ml}$ incubation bottles based on physiological proportions. Short chain fatty acids in fermentation fluid were estimated in duplicate by gas chromatography (FID) $)^{(7)}$.
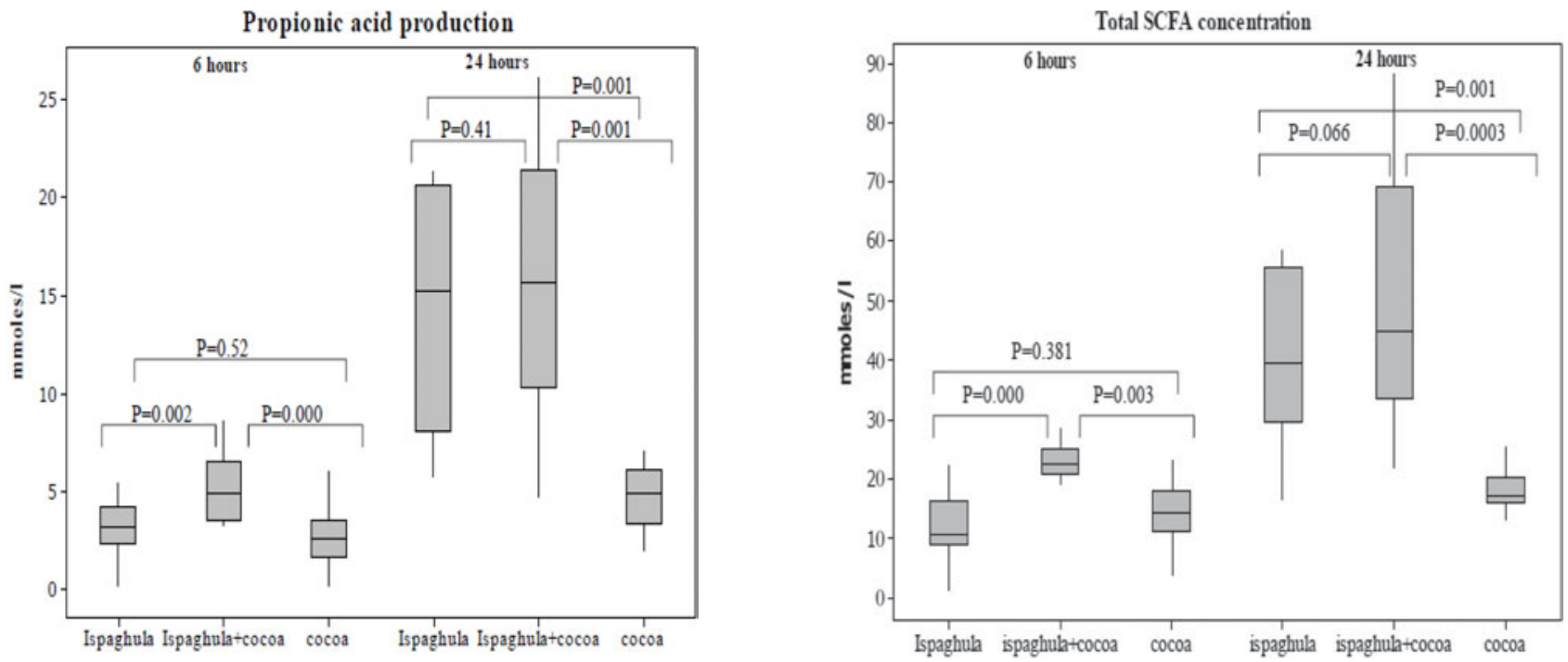

The fermentation of ispaghula (I) was as expected with a higher propionic acid production, albeit at a slower rate than cocoa (C). Thus propionic acid production was higher in the combined $\mathrm{C}+\mathrm{I}$ incubations than those containing $\mathrm{C}$ or I alone at $6 \mathrm{~h}$, however at $24 \mathrm{~h}$ no difference was observed when compared with I on its own (Figure). Similar results were observed for acetic acid, butyric acid and total short chain fatty acids although there was no significant difference between cocoa + ispaghula and cocoa for butyric acid. A predicted SCFA production was calculated by assuming the interaction of the fermentation of the ispaghula and fibre in cocoa was additive and compared to the actual SCFA of the mixture. There was no difference between predicted and actual values for the ispaghula + cocoa combination. This data does not support any inhibitory effect of cocoa polyphenolics on fermentation of ispaghula.

1. Jaganath IB, Mullen W, Lean MEJ et al. (2009) Free Radical Biology and Medicine 47, 1180-1189.

2. Saura-Calixto F, Perez-Jimenez J, Tourino S et al. (2010) Molecular Nutrition and Food Research 54, 939-943.

3. Kurek A, Grudniak AM, Kraczkiewicz-Dowjat A et al. (2011) Polish J of Microbiology 60, 3-12.

4. Cushnie T \& Lamb AJ (2011) Int J of Antimicrobial agents 38, 99-107.

5. Juskiewicz J, Zary-Sikorska E, Zdunczyk Z et al. (2011) Archives of Animal Nutr 65, 74-87.

6. Edwards CA, Gibson G, Champ M et al. (1996) J Sci Food Agric 71, 209-217.

7. Laurentin A, Edwards CA (2004) Eur J Clin Nutr 43, 183-189. 